\title{
A modelling framework for the olfactory system of the honeybee using GeNN (GPU enhanced Neuronal Network simulation environment)
}

\author{
Esin Yavuz, Thomas Nowotny ${ }^{*}$ \\ From 1st International Workshop on Odor Spaces \\ Hannover, Germany. 4-7 September 2013
}

We present an example implementation of a minimal model of honeybee olfactory system on massively parallel GPU hardware using GPU-specific code generation with GeNN[1]. This will be a first step to provide a physiologically coherent model of the honeybee olfactory system to be implemented in real time on fying autonomous robots for the "Green Brain Project".

The "Green Brain Project" will combine computational neuroscience modelling, learning and decision theory, modern parallel computing methods and robotics with data from state-of-the-art neurobiological experiments on cognition in the honeybee Apis mellifera, to build and deploy a modular model of the honeybee brain describing detection, classification and learning in the olfactory and optic pathways as well as multi-sensory integration across these sensory modalities. Unlike other brain models, which use expensive traditional supercomputing resources, the 'Green Brain' will be implemented on massively parallel, but affordable GPU technology. The 'Green Brain' will be deployed for the real-time control of a flying robot able to sense and act autonomously. This robot testbed will be used to demonstrate the development of new biomimetic control algorithms for artificial intelligence and robotics applications.

The objective for modelling olfaction in the "Green Brain Project" will extend previous attempts to model the antennal lobes and their constituent glomeruli (which encode olfactory cues), the projection neurons and the mushroom bodies. Odours are known to have a distributed representation in the antennal lobe, encoded as differential activation levels of glomerular populations. Odour mixtures are represented as a non-trivial combination of constituent odours' representations, and formation of long-term memories associated with such odour mixtures has been shown to induce volume changes in glomeruli indicating a cross-inhibitory effect between neural codings [2]. The modelling will also consider how mechanisms might implement known classification rules, such as in the models of insect olfactory classification by Huerta et al. [3] and Nowotny et al. [4].

In this study, we present some benchmarking results. We perform performance and scalability tests on an NVIDIA Tesla C2070 GPU with an Intel ${ }^{\circledR}$ Xeon(R) E5-2609 CPU running Ubuntu 12.04 LTS. Preliminary results show that as the network size increases, GPU simulations outperform CPU simulations. We suggest that implementation of realistic neural networks using GPUs will make real-time simulations of natural-like perception more efficient which would then provide a better understanding of sensory processing.

Published: 16 April 2014

References

1. The GeNN Project., URL http://genn.sourceforge.net.

2. Hourcade BB, Perisse E, Devaud J-M, Sandoz J-C: Long-term memory shapes the primary olfactory center of an insect brain. Learn Mem 2009, 16:607-615.

3. Huerta R, Nowotny T, Garcia-Sanchez M, Abarbanel HDI, Rabinovich M: Learning classification in the olfactory system of insects. Neural Comput 2004, 16:1601-1640.

4. Nowotny T, Huerta R, Abarbanel HDI, Rabinovich MI: Self-organization in the olfactory system: one shot odor recognition in insects. Biol Cybern 2005, 93:436-446.

doi:10.1186/2044-7248-3-S1-P23

Cite this article as: Yavuz and Nowotny: A modelling framework for the olfactory system of the honeybee using GeNN (GPU enhanced Neuronal Network simulation environment). Flavour 2014 3(Suppl 1):P23. 